\title{
A Simple Distributed Method for Relay Selection in Cooperative Diversity Wireless Networks, based on Reciprocity and Channel Measurements
}

\author{
Aggelos Bletsas, Andrew Lippman and David P. Reed \\ Massachusetts Institute of Technology \\ Media Laboratory \\ 20 Ames St, Cambridge MA 02139
}

\begin{abstract}
We propose a simple distributed method to find out the "best" end-to-end path between source and destination, among $M$ possible relays. The method requires no explicit communication among the relays, assumes no prior knowledge of network geometry and is based on instantaneous wireless channel measurements and reciprocity. The success (or failure) to select the "best" available path, depends on the statistics of the wireless channel, and a methodology to evaluate performance for any kind of wireless channel statistics, is provided. Benefits of cooperative diversity are increased with increased number of cooperating relays, even though a single relay transmits. The method simplifies required space-time coding and coordination among the cooperating terminals.
\end{abstract}

\section{INTRODUCTION}

The inherently broadcast nature of the wireless medium is exploited in cooperative diversity transmission schemes, where the destination combines direct transmission from a source with relayed transmissions from a number of overhearing terminals. The latter are willing to cooperate since they are using their own battery energy to relay information, originated from and destined to different users. Cooperative diversity leads to increased robustness against wireless fading, at the final destination.

Half-duplex schemes with a single relay between source and destination [8] or multiple relays [9] have been proposed, where the source transmits during the first phase of the scheme and the relay(s) transmit simultaneously during the second phase of the scheme, a version of what they received. Increased performance is achieved when the source transmits a different information symbol during the second phase [1], [10], or when direct and relayed transmissions occur within the same symbol period, when special signal modulation is used, in combination with oversampling [3].

When more than one relays are used, special modulation and error correction techniques are needed (also known as spacetime coding). Moreover, coordination among the cooperating terminals is required that would allow them to form cooperation groups and transmit simultaneously during the second

This work was supported by NSF under grant number CNS-0434816, the MIT Media Laboratory Digital Life Program and a Nortel Networks graduate fellowship award. phase. Space-time coding and coordination for more than one relay is still an open and fruitful topic of research [9].

In this work, we take a radically different approach to the problem of efficient cooperative diversity with more than one cooperating relays $(M \geq 2)$. Instead of trying to find a space-time code for $M \geq 2$ that could scale in practice, we devise a simple, distributed scheme to select a single relay out of $M$ candidates that has the best "signal path" from source to relay as well as relay to destination. The scheme requires no prior knowledge on network topology, is based on instantaneous channel measurements and assumes no global knowledge of the wireless channel conditions among all cooperating terminals. Therefore, the method is applicable for distributed implementation, even in environments where one relay might be "hidden" from the others.

The method simplifies space-time coding, since a single "best" relay transmits during the second phase. At the same time, the method explicitly addresses coordination among the cooperating terminals. Our approach could be implemented in any channel access scheme and a demonstration of the proposed scheme has been constructed in our lab, using low-cost, embedded radios [6]. In this work, a simple analysis of the algorithm is provided. Given the dependence of the selection scheme on the wireless channel conditions, performance is evaluated according to the statistics of the wireless channel. The analytical solution applies for any kind of wireless channel distribution and a specific example on Rayleigh fading is given.

\section{Method of Distributed Timers}

According to opportunistic relaying, a single relay among a set of $M$ nodes is selected, depending on which relay provides for the "best" end-to-end path between source and destination (figure 1). The wireless channel $a_{s i}$ between source and each relay $i$, as well as the channel $a_{i d}$ between relay $i$ and destination, affect performance. Since communication among all relays should be minimized for reduced overall overhead, a method based on time was selected: each relay $i$ "feeds" a timer with a parameter $h_{i}$ based on the channel conditions $a_{s i}, a_{i d}$. The timer of the relay with the best channel conditions 


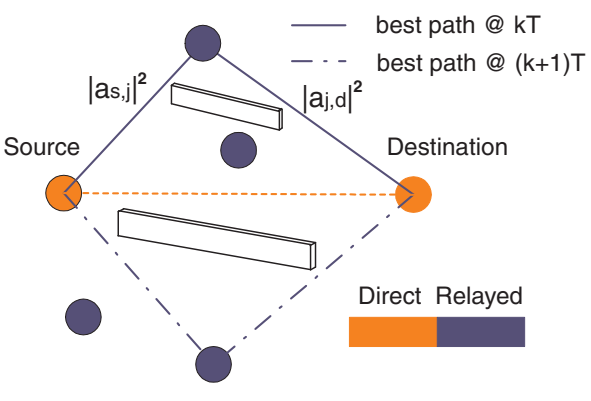

Fig. 1. Source transmits to destination and neighboring nodes overhear the communication. The relay with the "best" end-to-end path among M candidates is selected to relay information, via a distributed mechanism based on instantaneous channel measurements.

will expire first. All relays, while waiting for their timer to reduce to zero (i.e. to expire) are in listening mode. As soon as they hear another relay forwarding information (the best relay), they back off.

For the case where all relays can listen source and destination, but they are "hidden" from each other (i.e. they can not listen each other), the best relay could notify the destination with a short duration flag packet and the destination could then notify all relays with a short broadcast message.

All the above assume that all relays start their timers at the same time. Synchronization can be easily achieved by the exchange of Ready-to-Send (RTS) and Clear-to-Send (CTS) packets between source and destination. Relays can start their timers as soon as they receive the CTS packet. In that case, synchronization error on the order of propagation delay differences across all destination-relay pairs should be taken into account. For the cases where source and destination are not in direct range, they need to synchronize their RTS/CTS exchange by other means. For example, Network Time Keeping algorithms in client/server setups, such as those examined in [2] could be employed. Or fully decentralized solutions for network time keeping could be facilitated, such as those demonstrated in [4].

The RTS/CTS mechanism, existent in most MAC protocols, is also necessary for channel estimation at the relays: the transmission of RTS from the source allows for the estimation of the wireless channel $a_{s i}$ between source and relay $i$, at each relay $i$. Similarly, the transmission of CTS from the destination, allows for the estimation of the wireless channel $a_{i d}$ between relay $i$ and destination, at each relay $i$, according to the reciprocity theorem.

The channel estimates $a_{s i}, a_{i d}$ at each relay, describe the quality of the wireless path source-relay-destination, for each relay. Opportunistic relaying is about selecting the "best" path among $M$ possible options. Since the two hops are equally important for end-to-end performance, each relay should quantify its appropriateness as an active relay, using a function that balances the two hops. Two functions are used in this work. Under policy I, the minimum of the two is selected (equation (1)), while under policy II, the harmonic mean of the two is used (equation (2)).

Under policy I:

$$
h_{i}=\min \left\{\left|a_{s i}\right|^{2},\left|a_{i d}\right|^{2}\right\}
$$

Under policy II:

$$
h_{i}=\frac{2}{\frac{1}{\left|a_{s i}\right|^{2}}+\frac{1}{\left|a_{i d}\right|^{2}}}=\frac{2\left|a_{s i}\right|^{2}\left|a_{i d}\right|^{2}}{\left|a_{s i}\right|^{2}+\left|a_{i d}\right|^{2}}
$$

The relay that maximizes function $h_{i}$ is the one with the "best" end-to-end path between initial source and final destination (equation (4)). All relays will start their timer with an initial value, inversely proportional to the end-to-end channel quality $h_{i}$, according to the following equation:

$$
X_{i}=\frac{\lambda}{h_{i}}
$$

$\lambda$ is a constant that converts units of channel quality $|a|^{2}$ in units of time. It has units "unit of time" $x$ "unit of $|a|^{2}$ ". For example, if time is measured in $\mu$ secs and $|a|^{2}$ in units of power, then $\lambda$ could have values in $\mu$ sec $\mu W$ atts.

Assuming synchronization among the relays (either from the RTS/CTS exchange or through explicit multi-hop schemes as explained above), all relays start their timer simultaneously, with different initial values, depending on their channel realizations. The "best" relay is the one with its timer reduced to zero first (since it started from a smaller initial value, according to equations (3), (5). This is the relay $b$ that will participate in forwarding information. The rest of the relays, will back off.

$$
\begin{aligned}
h_{b} & =\max \left\{h_{i}\right\}, \Longleftrightarrow \\
X_{b} & =\min \left\{X_{i}\right\}, i \in[1 . . M] .
\end{aligned}
$$

In the following section, we will quantify the probability any other relay's timer (apart from the "best" relay) to expire within the same interval $c$, where the best relay timer expires. In that way, we will be able to calculate the probability this scheme to succeed in selecting the "best" relay.

As can be seen from the above equations, the scheme depends on the instantaneous channel realizations or equivalently, to received SNRs (assuming similar transmission power levels and noise floors in all terminals). Therefore, the best relay selection algorithm should be applied as often as the wireless channel changes. That rate of change depends on the coherence time of the channel. In the following section we will calculate the probability of successful relay selection, even at the case where the relays are hidden from each other.

\section{ANALYSIS}

The probability of having two or more relay timers expire "at the same time" is zero. However, the probability of having two or more relay timers expire within the same time interval $c$ is non zero and can be analytically evaluated, given knowledge of the wireless channel statistics. 


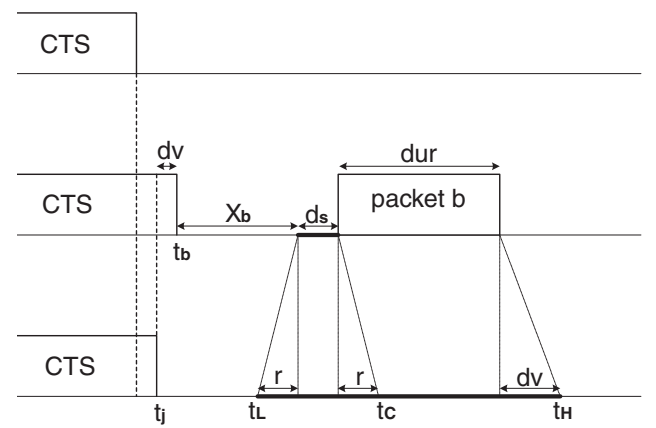

Fig. 2. The middle raw corresponds to the "best" relay. Other relays (top or bottom raw) could erroneously be selected as "best" relays, if their timer expired within intervals when they can not hear the best relay transmission. That can happen in the interval $\left[t_{L}, t_{C}\right]$ for case (a)(No Hidden Relays) or $\left[t_{L}, t_{H}\right]$ for case (b)(Hidden Relays).

The only case where opportunistic relay selections fails, is when the relays cannot listen each other and therefore, one relay can not detect that another relay is more appropriate for information forwarding. Note that we have already assumed that all relays can listen initial source and destination (figure 1), otherwise they do not participate in the scheme. We will assume two extreme cases: a) all relays can listen to each other b) all relays are hidden from each other (but they can still listen source and destination). In the second case, the best relay sends a flag packet to destination (or source) to notify for its candidacy, as the best relay. Then the destination (or source) notifies all relay nodes with a short broadcast message.

From figure 2, collision of two or more relays can happen if the best relay timer $X_{b}$ and one or more other relays expire within $\left[t_{L}, t_{C}\right]$ for case (a), or within $\left[t_{L}, t_{H}\right]$ for case (b). In any case, the collision probability can be upper bounded by the following expression:

$$
\begin{aligned}
\operatorname{Pr}(\text { Collision }) & \leq \operatorname{Pr}\left(\text { any } X_{j}<X_{b}+c \mid j \neq b\right) \\
\text { where } X_{b} & =\min \left\{X_{j}\right\}, j \in[1, M] \text { and } c>0 .
\end{aligned}
$$

and

(a) No Hidden Relays:

$$
c=r_{\max }+\left|v_{b}-v_{j}\right|_{\max }+d_{s}
$$

(b) Hidden Relays:

$$
c=r_{\max }+2\left|v_{b}-v_{j}\right|_{\max }+d_{s}+d u r
$$

- $v_{j}$ : propagation delay between relay $j$ and destination.

- $r_{\max }$ : maximum propagation delay between two relays.

- $d_{s}$ : receive-to-transmit switch time of each radio.

- dur: duration of flag packet, transmitted by "best" relay.

The upper bound in (6) and equations (7), (8) can be easily derived taking into account propagation delays, radio switch time and flag packet duration.

In the following section, we will provide an analytic way to calculate the upper bound of equation (6). But before doing so, we can easily show that this probability can be made arbitrary small, close to zero.
If $X_{b}=\min \left\{X_{j}\right\}, j \in[1, M]$ and $Y_{1}<Y_{2}<\ldots<Y_{M}$ the ordered random variables $\left\{X_{j}\right\}$ with $X_{b} \equiv Y_{1}$, then:

$$
\operatorname{Pr}\left(\text { any } X_{j}<X_{b}+c \mid j \neq b\right)=\operatorname{Pr}\left(Y_{2}<Y_{1}+c\right)
$$

Given that $Y_{j}=\lambda / h_{(j)}, Y_{1}<Y_{2}<\ldots<Y_{M}$ is equivalent to $1 / h_{(1)}<1 / h_{(2)}<\ldots<1 / h_{(M)}$, equation (9) is equivalent to

$$
\operatorname{Pr}\left(Y_{2}<Y_{1}+c\right)=\operatorname{Pr}\left(\frac{1}{h_{(2)}}<\frac{1}{h_{(1)}}+\frac{c}{\lambda}\right)
$$

and $Y_{1}<Y_{2}<\ldots<Y_{M} \Leftrightarrow h_{(1)}>h_{(2)} \ldots>h_{(M)}(h, \lambda, c$ are positive numbers).

From the last equation, it is obvious that increasing $\lambda$ at each relay (in equation (3)), we can reduce the probability of collision to zero since the upper bound of (10) goes to zero with increasing $\lambda$.

In practice, $\lambda$ can not be made arbitrarily large, since it also "regulates" the expected time, needed for the network to find out the "best" relay. From equation (3) and Jensen's inequality we can see that

$$
E\left[T_{j}\right]=E\left[\lambda / h_{j}\right] \geq \lambda / E\left[h_{j}\right]
$$

or in other words, the expected time needed for each relay to flag its presence, is lower bounded by $\lambda$. Therefore, there is a trade-off between probability of collision and speed of relay selection. We need to make $\lambda$ as big as possible to reduce collision probability and at the same time, as small as possible, to quickly select the best relay, before the channel changes again (i.e. within the coherence time of the channel).

In the following section we provide a method to quantify performance for any kind of wireless channel statistics and any kind of values for $c$ and $\lambda$ and show that the scheme can perform reasonably well.

\section{A. Calculating $\operatorname{Pr}\left(Y_{2}<Y_{1}+c\right)$}

Theorem 1: The joint probability density function of the minimum and second minimum among $M$ i.i.d. positive random variables $X_{1}, X_{2}, \ldots, X_{M}$, each with probability density function $f(x)$ and cumulative distribution function $F(x)$, is given by the following equation:

$$
\begin{aligned}
f_{Y_{1}, Y_{2}}\left(y_{1}, y_{2}\right)= & M(M-1) f\left(y_{1}\right) f\left(y_{2}\right)\left[1-F\left(y_{2}\right)\right]^{M-2} \\
& 0<y_{1}<y_{2}, \\
f_{Y_{1} Y_{2}}\left(y_{1}, y_{2}\right)= & 0 \\
& \text { elsewhere. }
\end{aligned}
$$

where $Y_{1}<Y_{2}<Y_{3} \ldots<Y_{M}$ are the $M$ ordered random variables $X_{1}, X_{2}, \ldots, X_{M}$.

Proof: $f_{Y_{1}, Y_{2}}\left(y_{1}, y_{2}\right) d y 1 d y 2=$ $\operatorname{Pr}\left(Y_{1} \in d y_{1}, Y_{2} \in d y_{2}\right)=$

$\operatorname{Pr}\left(\right.$ one $X_{i}$ in $d y_{1}$, one $X_{j}$ in $d y_{2}$ (with $y_{2}>y_{1}$ and $i \neq j)$, and all the rest $X_{i}^{\prime}$ s greater than $\left.y_{2}\right)=$

$$
=2\left(\begin{array}{c}
M \\
2
\end{array}\right) \operatorname{Pr}\left(X_{1} \in d y_{1}, X_{2} \in d y_{2}\left(y_{2}>y_{1}\right)\right. \text {, }
$$$$
\left.X_{i}>y_{2}, i \in[3, M]\right)=
$$ 


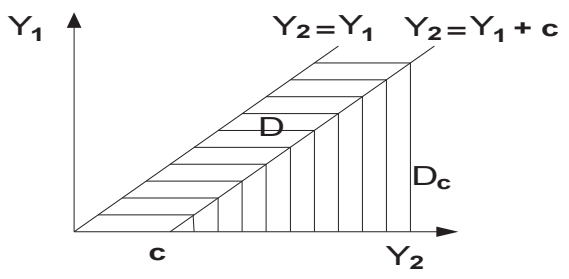

Fig. 3. Regions of integration of $f_{Y_{1}, Y_{2}}\left(y_{1}, y_{2}\right)$, for $Y_{1}<Y_{2}$ needed in Lemma I for calculation of $\operatorname{Pr}\left(Y_{2}<Y_{1}+c\right), c>0$.

$$
\begin{gathered}
=2\left(\begin{array}{c}
M \\
2
\end{array}\right) f\left(y_{1}\right) d y_{1} f\left(y_{2}\right) d y_{2}\left[1-F\left(y_{2}\right)\right]^{M-2}= \\
=M(M-1) f\left(y_{1}\right) f\left(y_{2}\right)\left[1-F\left(y_{2}\right)\right]^{M-2} d y_{1} d y_{2}, \\
\text { for } 0<y_{1}<y_{2} .
\end{gathered}
$$

The third equality is true since there are $\left(\begin{array}{c}M \\ 2\end{array}\right)$ pairs in a set of $\mathrm{M}$ i.i.d. random variables. The factor 2 comes from the fact that ordering in each pair matters, hence we have a total number of $2\left(\begin{array}{c}M \\ 2\end{array}\right)$ cases, with the same probability, assuming identically distributed random variables. That concludes the proof.

Using Theorem 1, we can prove the following lemma:

Lemma 1: Given $M$ i.i.d. positive random variables $X_{1}, X_{2}, \ldots, X_{M}$, each with probability density function $f(x)$ and cumulative distribution function $F(x)$, and $Y_{1}<$ $Y_{2}<Y_{3} \ldots<Y_{M}$ the $M$ ordered random variables $X_{1}, X_{2}, \ldots, X_{M}$, then $\operatorname{Pr}\left(Y_{2}<Y_{1}+c\right)$, where $c>0$, is given by the following equations:

$$
\begin{gathered}
\operatorname{Pr}\left(Y_{2}<Y_{1}+c\right)=1-I_{c} \\
I_{c}=M(M-1) \int_{c}^{+\infty} f(y)[1-F(y)]^{M-2} F(y-c) d y
\end{gathered}
$$

Proof: The joint pdf $f_{Y_{1}, Y_{2}}\left(y_{1}, y_{2}\right)$ integrates to 1 in the region $D \cup D_{c}$, as it can be seen in figure 3 . Therefore:

$$
\begin{aligned}
\operatorname{Pr}\left(Y_{2}<Y_{1}+c\right) & =\iint_{D} f_{Y_{1}, Y_{2}}\left(y_{1}, y_{2}\right) d y 1 d y 2 \\
& =1-\iint_{D_{c}} f_{Y_{1}, Y_{2}}\left(y_{1}, y_{2}\right) d y 1 d y 2 \\
& =1-I_{c}
\end{aligned}
$$

Again from figure $3, I_{c}$ can easily be calculated:

$$
\begin{gathered}
I_{c}= \\
\int_{y_{2}=c}^{+\infty} f\left(y_{2}\right)\left[1-F\left(y_{2}\right)\right]^{M-2} \int_{0}^{y_{2}-c} f\left(y_{1}\right) d y_{1} d y_{2} \\
=M(M-1) \int_{y_{2}=c}^{+\infty} f\left(y_{2}\right)\left[1-F\left(y_{2}\right)\right]^{M-2} F\left(y_{2}-c\right) d y_{2} \quad(15)
\end{gathered}
$$

The last equation concludes the proof.

\section{RESULTS}

Using theorem 1 and lemma 1 of the previous section, we can quantify $\operatorname{Pr}\left(Y_{2}\right)<Y_{1}+c$, for any kind of wireless channel statistics. From the above, we have restricted discussion to identically distributed wireless channel realizations. The results could be extended to the non-identically distributed case, where geometry is taken into account. We chose to restrict the discussion to the identically distributed case for simplicity and leave the non-identical (but still independent) case for future work. In the numerical results below, we have normalized $E\left[\left|a_{s i}\right|^{2}\right]=E\left[\left|a_{i d}\right|^{2}\right]=E\left[|a|^{2}\right]=1$.

According to theorem and lemma 1, we need to calculate the probability distribution of $X_{i}$ for $i \in[1, M]$. From equation (3) it is easy to see that the CDF and pdf of $X_{i}$ are related to the respective distributions of $h_{i}$ according to the following equations:

$$
\begin{gathered}
F(x) \equiv C D F_{X_{i}}(x)=\operatorname{Pr}\left\{X_{i} \leq x\right\}=1-C D F_{h_{i}}\left(\frac{\lambda}{x}\right) \\
f(x) \equiv p d f_{X_{i}}(x)=\frac{d}{d x} F(x)=\frac{\lambda}{x^{2}} p d f_{h_{i}}\left(\frac{\lambda}{x}\right)
\end{gathered}
$$

After calculating equations (16), (17), and for a given $c$ calculated from (7) or (8), we can calculate probability of collision using equation (13).

Before proceeding to special cases, we need to observe that for a given distribution of the wireless channel, collision performance depends only on the ratio $c / \lambda$, as can be seen from equation (10), discussed earlier.

\section{A. Rayleigh Fading}

Assuming $\left|a_{s i}\right|,\left|a_{j d}\right|$ are i.i.d according to Rayleigh distribution, for any $i, j \in[1, M]$, then $|a|^{2}$ is distributed according to an exponential distribution, with parameter $\beta$ (e.g. $\left.E\left[|a|^{2}\right]=1 / \beta\right)$.

Using the fact that the minimum of two i.i.d. exponentials is again an exponential with doubled parameter, we can calculate the distributions for $h_{i}$ under policy I (equation 1). For policy II (equation 2), the distributions of the harmonic mean, have been calculated analytically in [7]. Equations (16) and (17) under the above assumptions, become:

under policy I:

$$
\begin{aligned}
F(x) & =e^{-2 \beta \lambda / x} \\
f(x) & =\frac{2 \beta \lambda}{x^{2}} e^{-2 \beta \lambda / x}
\end{aligned}
$$

under policy II:

$$
\begin{aligned}
F(x) & =\frac{\lambda \beta}{x} e^{-\lambda \beta / x} K_{1}\left(\frac{\lambda \beta}{x}\right) \\
f(x) & =\frac{\lambda^{2}}{x^{3}} b^{2} e^{-\lambda \beta / x}\left[K_{1}\left(\frac{\lambda \beta}{x}\right)+K_{0}\left(\frac{\lambda \beta}{x}\right)\right]
\end{aligned}
$$

where $K_{i}(x)$ is modified Bessel function of the second kind and order $i$. 


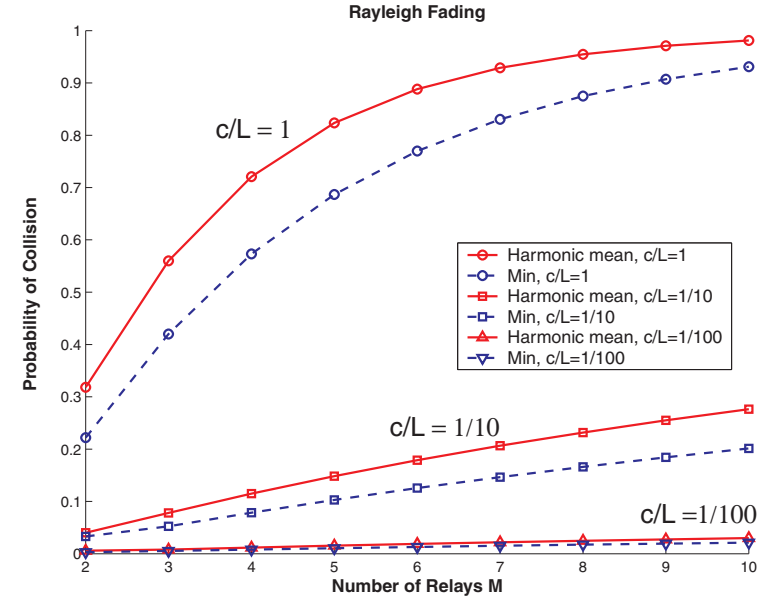

Fig. 4. Probability of collision for two policies, in Rayleigh Fading, as a function of number of relays $(M)$. Notice that probability can be made arbitrarily small with decreased ratio $c / \lambda$.

In figure 4, equation (13) is calculated for the tho policies. We can see that the collision probability can be made arbitrarily small, with decreased ratio $c / \lambda$. That practically means that the smaller the propagation delays among relays (or equivalently the smaller the tx range of the radios) and the faster the radios used (for smaller duration in time of the flag packet), the better performance. Practically, for $c \approx$ $1 \mu \mathrm{sec}$, corresponding to $802.11 \mathrm{~b}$ tx range, and average time needed for relay selection, on the order of 100 usecs (or $\lambda \approx 100 \mu$ secs units of $h_{i}$ ), the collision probability can drop below $1 \%$.

Another interesting observation is that the two policies (harmonic mean vs minimum of the two wireless hops) provide similar results. Analysis for Ricean Fading has been excluded due to space restrictions and could be found in [5], [6].

\section{CONCLUSION}

We proposed a simple method to select the appropriate end-to-end path between a source and a destination, among a set of $M$ cooperating terminals. The method is distributed and requires no global knowledge of the wireless channel conditions across all possible paths, since each relay needs to know only its own wireless channel toward the source and the destination. Distributed selection is achieved through an access scheme based on distributed timers and channel estimation using reciprocity.

This work provided a method to calculate the performance of the relay selection algorithm, for any king of wireless fading model. An example for the case of Rayleigh fading was provided, showing reasonable performance.

The proposed method addresses coordination among the cooperating terminals and simplifies space-time coding since the "best" relay is selected to retransmit, instead of allowing all relays to simultaneously relay. It can be shown that the diversity of the scheme in on the order of $M$, even though one relay transmits [5], [6]. Benefits of cooperative diversity (figure

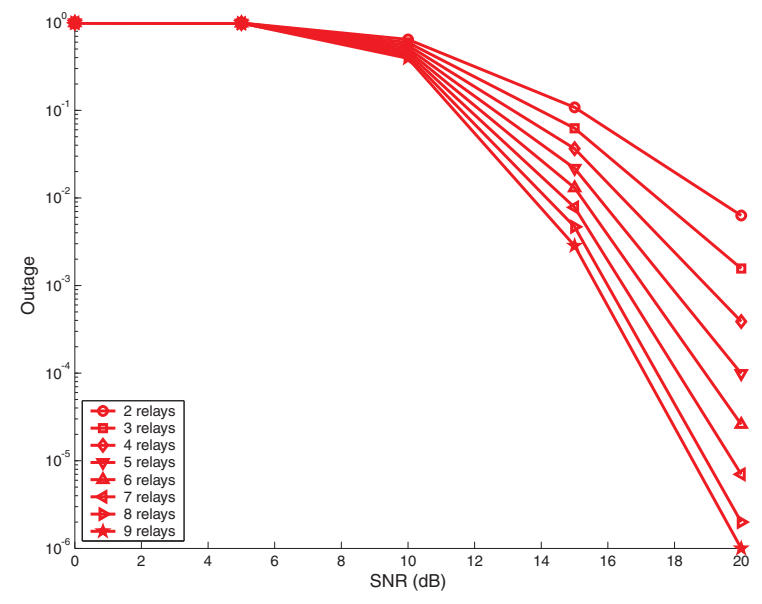

Fig. 5. Outage probability when "best" relay is selected for various cases of number of relays $M$. Rate is at $2 \mathrm{bps} / \mathrm{Hz}$ and Rayleigh fading is used with the characteristics described in section IV. Notice that a single relay out of $M$ transmits.

5) could be realized in practice using simple radio software and hardware [6].

\section{ACKNOWLEDGMENT}

The authors would like to thank Wei Chai from Media Lab's Music, Mind and Machine Group.

\section{REFERENCES}

[1] K. Azarian, H. El Gamal, P. Schniter, "On the Achievable DiversityMultiplexing Tradeoff in Half-duplex Cooperative Channels", submitted for publication to IEEE Transactions of Information Theory, available at http://www.ece.osu.edu/ schniter/research.html

[2] A. Bletsas, "Evaluation of Kalman Filtering for Network Time Keeping”, Proceedings of IEEE First International Conference on Pervasive Computing and Communications (PerCom 2003), pp. 289-296, Dallas-Fort Worth Texas, March 23-26, 2003.

[3] A. Bletsas, A. Lippman, "Efficient Collaborative (Viral) Communication in Ofdm based Wlans”, Proceeding of ISART 2003, pp. 27-32, Boulder Colorado, March 2003.

[4] A. Bletsas, A. Lippman, "Spontaneous Synchronization in Multi-hop Embedded Sensor Networks: Demonstration of a Server-free Approach", Second European Workshop on Wireless Sensor Networks, February 2005.

[5] A. Bletsas, A. Khisti, D.P. Reed, A. Lippman, "A Simple Cooperative Diversity Method based on Network Path Selection", submitted for publication, IEEE Journal on Selected Areas of Communication, Special Issue on 4G, January 2005.

[6] A. Bletsas, Intelligent Antenna Sharing and User Cooperation in Wireless Networks, Ph.D Dissertation in preparation, Massachusetts Institute of Technology, Spring 2005.

[7] M. O. Hasna, M.S. Alouini, "End-to-End Performance of Transmission Systems With Relays Over Rayleigh-Fading Channels”, IEEE Transactions on Wireless Communications, vol. 2, no. 6, November 2003.

[8] J. N. Laneman, D.N. Tse, G. W. Wornell, "Cooperative Diversity in Wireless Networks: Efficient Protocols and Outage Behavior”, submitted for publication to IEEE Transactions of Information Theory, available at http://www.nd.edu/ jnl/pubs/

[9] J. N. Laneman, G. W. Wornell, "Distributed Space-Time Coded Protocols for Exploiting Cooperative Diversity in Wireless Networks”, IEEE Trans. Inform. Theory, vol. 49, no. 10, pp. 2415-2525, Oct. 2003.

[10] A. Wittneben, B. Rankov, "Impact of Cooperative Relays on the Capacity of Rank-Deficient MIMO Channels", Proceedings of the 12th IST Summit on Mobile and Wireless Communications, Aveiro, Portugal, pp. 421-425, June 2003. 